\title{
A Disposable Multiplexed Chip for the Simultaneous Quantification of Key Parameters in Water Quality Monitoring
}

Huiyun Jiang ${ }^{\mathrm{a}}$, Bing Sun ${ }^{\mathrm{a}, *}$, Yan Jin ${ }^{\mathrm{b}}$, Junjie Feng ${ }^{\mathrm{b}}$, Hongwei Zhu ${ }^{\mathrm{a}}$, Lin Wang ${ }^{\mathrm{a}}$, Shucai Zhang ${ }^{\mathrm{b}}$, Zhe Yang

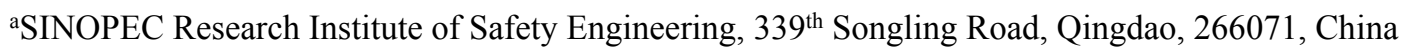

'State Key Laboratory of Safety and Control for Chemicals, 218 Yan'an $3^{\text {rd }}$ Road, Qingdao, 266071,

China 


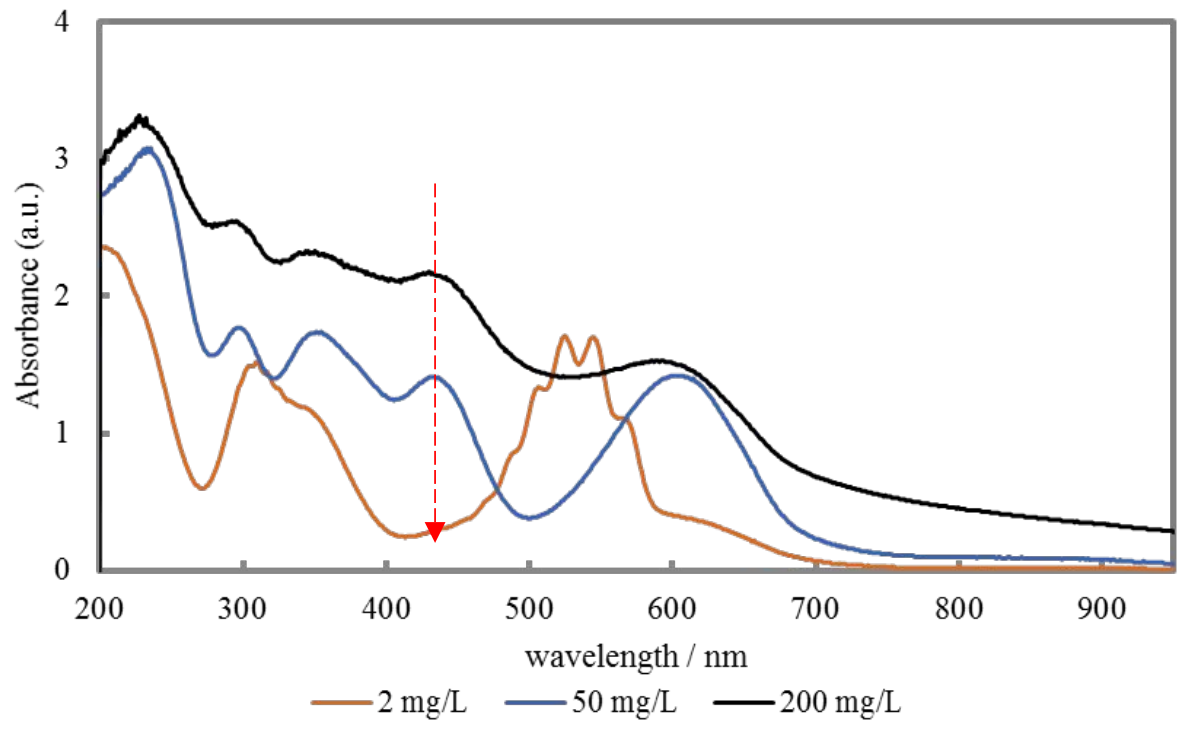

Figure S1 The absorbance spectrum of the detection solution after reaction at different COD concentrations 
Table S1 concentration of target molecules used in the cell phone imaging experiments

\begin{tabular}{lccccc}
\hline parameters & $\begin{array}{c}\text { nickel } \\
(\mathrm{mg} / \mathrm{L})\end{array}$ & $\begin{array}{c}\text { chromium } \\
(\mathrm{mg} / \mathrm{L})\end{array}$ & $\begin{array}{c}\mathrm{COD} \\
(\mathrm{mg} / \mathrm{L})\end{array}$ & $\begin{array}{c}\text { ammonia } \\
\text { nitrogen }(\mathrm{mg} / \mathrm{L})\end{array}$ & $\begin{array}{c}\text { phosphate } \\
(\mathrm{mg} / \mathrm{L})\end{array}$ \\
\hline chemicals & $\mathrm{Ni}\left(\mathrm{NO}_{3}\right)_{2}$ & $\mathrm{~K}_{2} \mathrm{Cr}_{2} \mathrm{O}_{7}$ & $\mathrm{KH}_{5} \mathrm{C}_{8} \mathrm{O}_{4}$ & $\left(\mathrm{NH}_{4}\right)_{2} \mathrm{SO}_{4}$ & $\mathrm{KH}_{2} \mathrm{PO}_{4}$ \\
$\begin{array}{c}\text { low } \\
\text { concentration } \\
\text { mid }\end{array}$ & 1 & 0.2 & 20 & 1 & 0.2 \\
$\begin{array}{c}\text { concentration } \\
\text { high }\end{array}$ & 5 & 1 & 100 & 5 & 1 \\
concentration & 10 & 2 & 200 & 10 & 2 \\
\hline
\end{tabular}


Table S2 standard substance and detection chemistry for each parameters

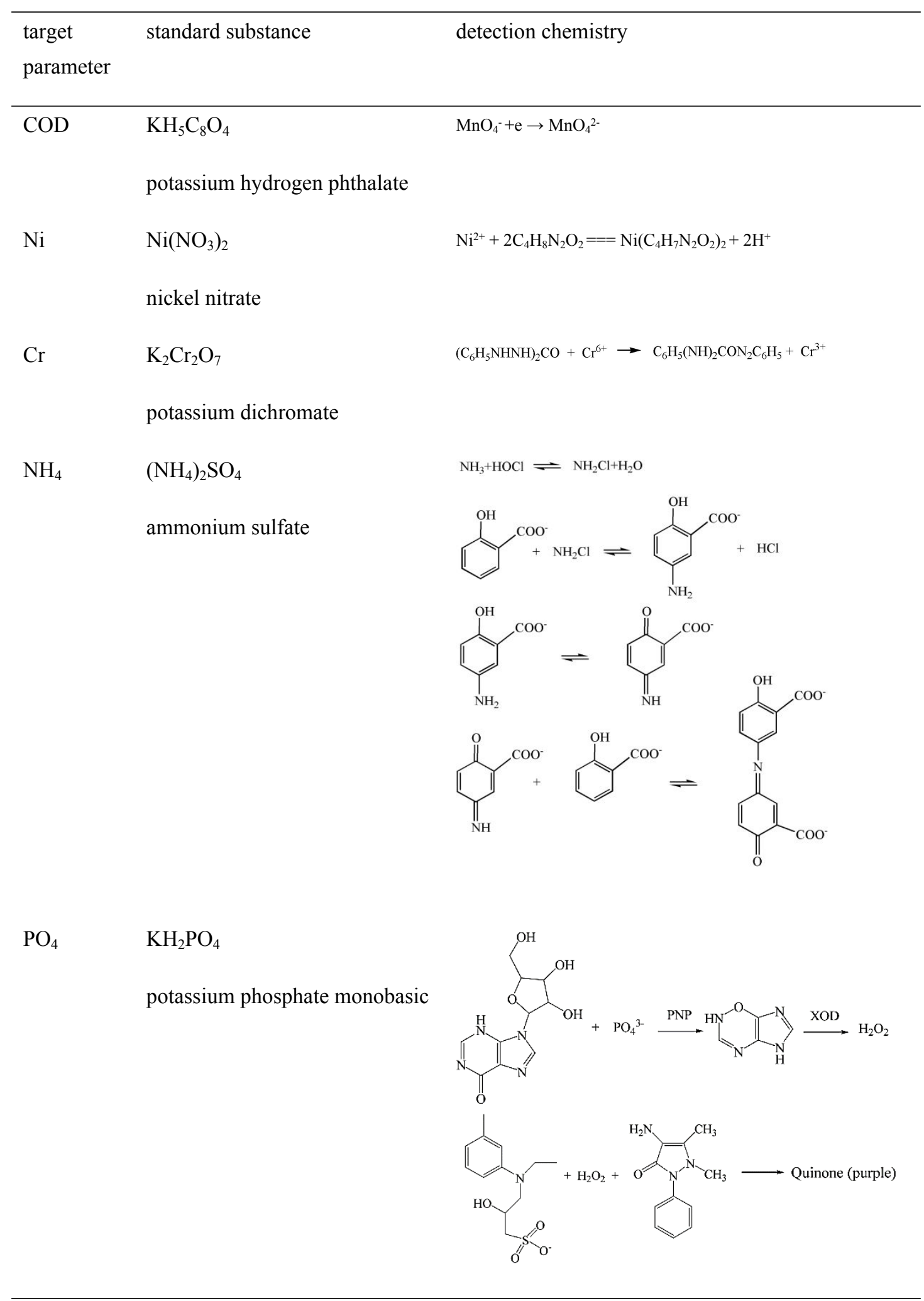


MATLAB code for $\mathrm{R}, \mathrm{G}$ and $\mathrm{B}$ value calculation

[filename,path]=uigetfile('*.jpg','load image files');

file=[path filename];

$i=$ imread(file);

$\operatorname{win}=[55]$;

img $=\mathrm{i}$;

figure;imshow(img);

$x=\operatorname{img}(:,:, 1)$;

$\mathrm{y}=\operatorname{img}(:,:, 2)$

$\mathrm{z}=\operatorname{img}(:, ;, 3)$

$\mathrm{X}=$ medfilt2(x,win);

$\mathrm{Y}=$ medfilt2(y,win);

$\mathrm{Z}=$ medfilt2(z,win);

$\mathrm{i}=\operatorname{cat}(3, \mathrm{X}, \mathrm{Y}, \mathrm{Z})$;

imshow(i)

$\%[\mathrm{a}, \mathrm{b}]=$ ginput;

button $=1$;

$\mathrm{n}=0$;

$\mathrm{a}=[]$

$\mathrm{b}=[]$

hold on;

while button $==1 \%$;

[xi,yi,button] $=\operatorname{ginput}(1)$;

$\mathrm{n}=\mathrm{n}+1$

if $n>1$

plot([xi,a(n-1,1)], [yi,b(n-1,1)], '-rs','LineWidth',1,... 
'MarkerEdgeColor','k',,...

'MarkerFaceColor','g',,..

'MarkerSize',5);

else

plot(xi, yi, 's','LineWidth',2,...

'MarkerEdgeColor','k',,...

'MarkerFaceColor','g',,..

'MarkerSize',5);

end

$\mathrm{a}(\mathrm{n}, 1)=\mathrm{xi}$

$\mathrm{b}(\mathrm{n}, 1)=\mathrm{yi} ;$

end

$\mathrm{c}=\mathrm{a}(2,1)-\mathrm{a}(1,1) ;$

$\mathrm{d}=\mathrm{b}(2,1)-\mathrm{b}(1,1)$;

image $=\operatorname{imcrop}(i,[a(1,1) b(1,1)$ c d] $)$;

close all;

imager $=$ image $(:,:, 1)$;

imageg $=$ image $(:,:, 2)$;

imageb $=$ image $(:,:, 3)$;

Rmean=mean2(imager);

Gmean=mean2(imageg);

Bmean=mean2(imageb);

Rstd=std2(imager);

Gstd=std2(imageg);

Bstd=std2(imageb); 